\title{
Study on the Design and Size Effect of Scaled Model for Inflated Fabric Membrane
}

\author{
Fen Zhang ${ }^{1, a}$, Shiming Zhou ${ }^{1, b}$, Jiehong Yuan ${ }^{1, c}$ \\ ${ }^{1}$ School of Traffic \& Transportation Engineering, National University of Defense Technology, \\ Hunan 410072, China \\ agodiva_zhang@foxmail.com, bshiming.zhou@nudt.edu.cn, cyjh210048@163.com
}

\begin{abstract}
Inflatable fabric membrane has been typically used in aeronautics and astronautics, large-scale construction and other fields because of its advantages: light weight, low transportation costs, and ease of deployment and storage. However, a natural challenge is the accurate characterization of the mechanical properties of the materials such as the airbag, subjected to the anisotropy of the membrane, the interaction between the fabric and the inflation pressure. In this paper, to estimate the effective elastic constant, the biaxial tensile tests of fabric film were performed by considering the effect of inflation pressure, this and specimen size on the elastic constants was obtained. Based on the similitude theory, the reduction ratio of inflatable fabric is deduced, and the irreducible factor is analysed. The validity of theoretical derivation is verified by numerical simulation and experiment of woven airbeams loaded in four-point bending. The error of scaled model is evaluated, which provides an effective technique for large-scale inflatable structure test. Abundant research results have been achieved in the test method of fabric membrane.
\end{abstract}

Keywords: Inflated fabric membrane, constitutive properties, similitude theory, size effect, scaled-model error.

\section{Introduction}

Inflatable fabric membrane can form a certain structure or member as load-carrying once air is inflated in special airbags [1]. It has been typically used in aeronautics and astronautics, large-scale construction and other fields [2] because of their main advantages: light weight, low transportation costs, and ease of deployment and storage. Its popular carrying structure forms are: circle and oval-shape, arch and ring (inflated arch, inflated ring) and cylinder (airbeam), their main application is as shown in Fig 1.

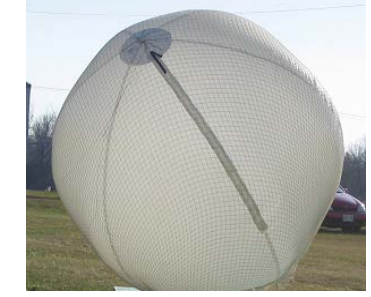

(a) Helium Balloon

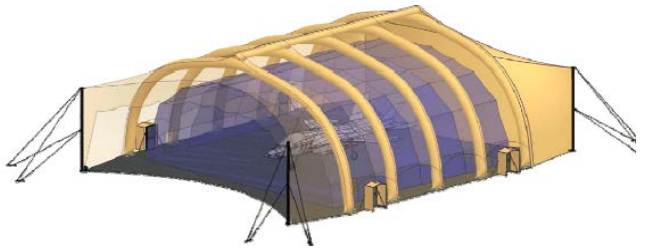

(b) Recreational Tents

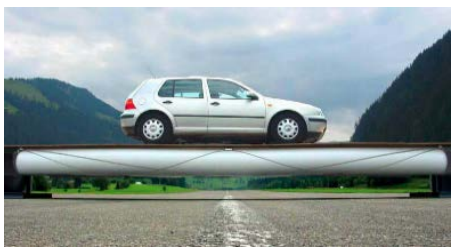

(c) Tensairity Bridge

Fig. 1 Bearing structure of balloon structure

The material of inflatable fabric membrane is laminated composite materials, the macroscopic material mechanical properties of anisotropy and non-linear etc. characteristic. Researches by Wicker [3] and Davids et al. [4] suggest that the axial and shear moduli of the fabric are strongly dependent on the inflated pressure and pre-tensioning of the fabric. Jean Paul [5] first treated fabric film as a structural material, to characterize the elastic modulus of a particular fabric by considering the pressure between the impact and the weft yarn Interaction, to assess the structural capacity of air beams to find a correct way. 
By the conditions of ground test, it is difficult to operate full-scale prototype test, thus scaled model test becomes the singly option. The developing scaled model study on inflatable fabric membrane facilitates engineering application like reduce costs, improve reliability and assess inflatable structure manufacturing process. Due to dimensional analysis method is simple and easy for engineering staff to understand, it becomes a preference for scaled model design.

The design of scaled model of inflatable membrane structure is different from the traditional one due to the following three issues: (1)The thickness of the membrane is much smaller than the size of the other direction, the thickness direction is difficult to shrink; (2)As laminated composite fabric material, inflatable membrane has anisotropic characteristics, and it's mechanical properties are subjected to size effect; (3)The exact response about the relationship between stiffness and pressure should be considerate. In 1998, Greschik introduced constant thickness scaling that involves a uniform scaling of all global dimensions of a pressurized membrane but no reduction of film thicknesses, subsequently carried out a number of studies [6-8]. Over the past decade, the research and application of inflatable scaled models mainly emphasize the spatial inflatable structures such as solar panels and antennas. Lienard et al. [9-14] showed that the scaled-model test guided by similarity criteria of constant thickness inflatable membrane for pneumatic structure can accurately simulate the structural response of the weightlessness space. Due to above research objects are isotropic, the constitutive model of linear elastic nonwoven fabric material is mature, and influence of structural size effect is not obvious. Nevertheless, the studies still laid the theoretical basis for the design of inflatable fabric membrane scaled model. This paper aims to study scaled model design and size effect on the basis of the fabric material inflatable structure.

\section{The method and test}

\subsection{The biaxial tensile/ torsion tests of fabric}

The influence of inflation pressure on fabric response was evaluated. Cylindrical biaxial test and inflatable tube test were proposed in this paper to measure membrane tension and shear modulus, as outlined in Fig 2.

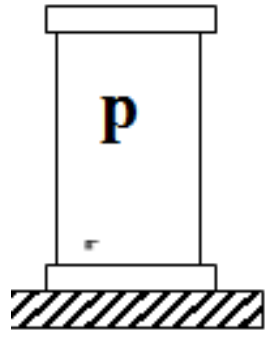

(a) Biaxial tensile test method
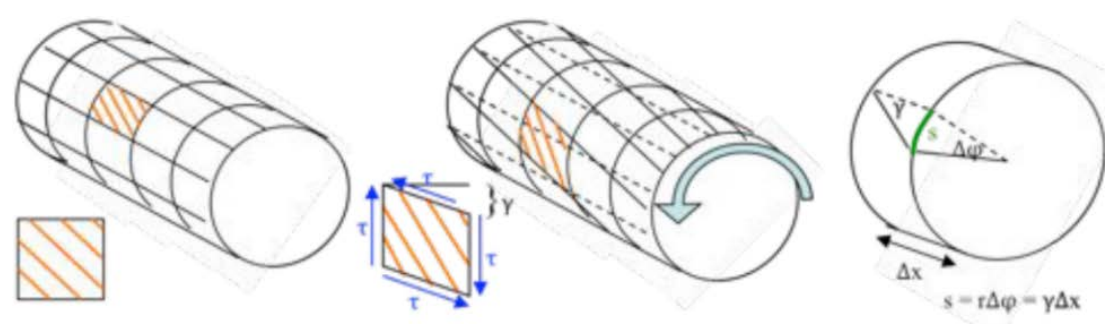

(b) Torsion test method

Fig. 2 Test program for inflatable pipes

The experimental setup used for both tension and torsion testing are shown in Fig.3. The specimens were made of QT-90S9198 polyester fiber membrane for three dimensions of the fabric tube, length $700 \mathrm{~mm}$, dia. $100 \mathrm{~mm}$, dia. $200 \mathrm{~mm}$ and dia. $300 \mathrm{~mm}$ respectively. According to the material strength, $1 / 4$ strength criteria design charge the load was conducted. The specific experimental design is shown in Table1.Experimental program is divided into two groups: (1) axial load test, axial and circumferential stress ratio from 1: 2 to 2: 1, to evaluate the impact of load ratio on the elastic modulus; by filling the specimen with different pressures, the assessment of size effect on the elastic modulus was evaluated by testing the specimens in different diameters. (2) Torsional load test, the specimens were subjected to torsion when the axial load was fixed, and the shear modulus of the material under different pressures and torsional and torsional loads was investigated. 


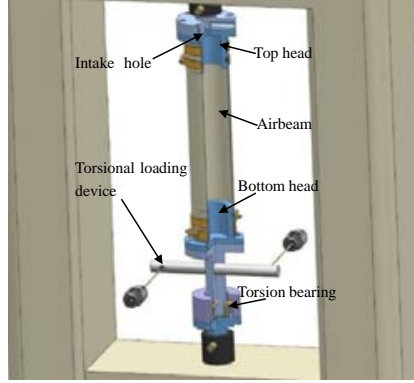

(a) Biaxial tension torsion testing device

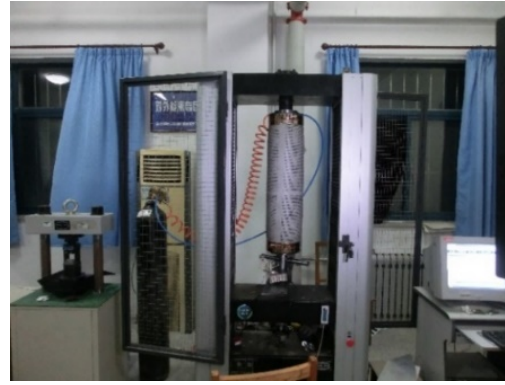

(b) Fabric tube specimen

Fig. 3 Test device and specimen for fabric

Table 1 Biaxial tensile and torsion test design table

\begin{tabular}{|c|c|c|c|c|c|c|c|c|c|}
\hline Test numble & 1 & 2 & 3 & 4 & 5 & 6 & 7 & 8 & 9 \\
\hline Specimen diameter (mm) & 100 & 100 & 100 & 200 & 200 & 200 & 300 & 300 & 300 \\
\hline Pressure (Kpa) & 100 & 200 & 400 & 60 & 100 & 200 & 60 & 90 & 120 \\
\hline
\end{tabular}

\subsection{Test Data Processing Method}

The membrane is assumed to be an orthotropic elastic material and elastic symmetry axis of orthogonal membrane. Regardless of shear, $N_{X} . N_{Y}$ are external loads of wrap and fill respectively; $\varepsilon_{x}, \varepsilon_{y}$ are strain of two directions. In accordance with the symmetry of the flexibility and stiffness matrices, the flexibility matrix of the orthotropic elastic fabric membrane is computed according to:

$$
\left[\begin{array}{ccc}
\sum_{i=1}^{n} N_{X i}^{2} & \sum_{i=1}^{n} N_{X i} N_{Y i} & 0 \\
\sum_{i=1}^{n} N_{X i} N_{Y i} & \sum_{i=1}^{n}\left(N_{X i}^{2}+N_{Y i}^{2}\right) & \sum_{i=1}^{n} N_{X i} N_{Y i} \\
\sum_{i=1}^{n} N_{X i} N_{Y i} & \sum_{i=1}^{n} N_{Y i}^{2}
\end{array}\right]\left\{\begin{array}{l}
E_{11} \\
E_{12} \\
E_{22}
\end{array}\right\}=\left\{\begin{array}{c}
\sum_{i=1}^{n} N_{X i} \varepsilon_{X i} \\
\sum_{i=1}^{n}\left(N_{Y i} \varepsilon_{X i}+N_{X i} \varepsilon_{Y i}\right) \\
\sum_{i=1}^{n} N_{Y i} \varepsilon_{Y i}
\end{array}\right\}
$$

Finally, the elastic modulus and Poisson's ratio are obtained from $E_{11}, E_{12}, E_{22}$ [15].

Treat the specimen as a beam, the shear modulus of the film can be calculated according to Eqs. (5):

$$
\begin{gathered}
\tau=\frac{T}{A \cdot r}=\frac{T}{2 \pi r t \cdot r}=\frac{T}{2 \pi r^{2} t} \\
\gamma=\frac{r \phi}{L} \\
G=\frac{\tau}{\gamma}
\end{gathered}
$$

Where $\mathrm{T}$ is torque; $\mathrm{A}$ is the cross-sectional area of the inflatable; $\phi$ is the torsion angle; $\mathrm{L}$ is the length of the tenders; $t$ is the thickness of membrane; $G_{x y}$ is the shear modulus from Eqs. (3)-(5).

\subsection{Biaxial tensile test results analysis}

Specimens with dia. $100 \mathrm{~mm}$, dia. $200 \mathrm{~mm}$ were subjected to three cycles of loading and unloading to obtain the stable axial stress and strain response at $100 \mathrm{KPa}$, so that the performance of air bags and fabrics achieves stability. According to the test results, the least square method is applied to obtain the fiber membrane elastic constant, Fig. 4 shows that the test method above is obviously affected by size effect, meanwhile, pressure and size have coupling effect. 


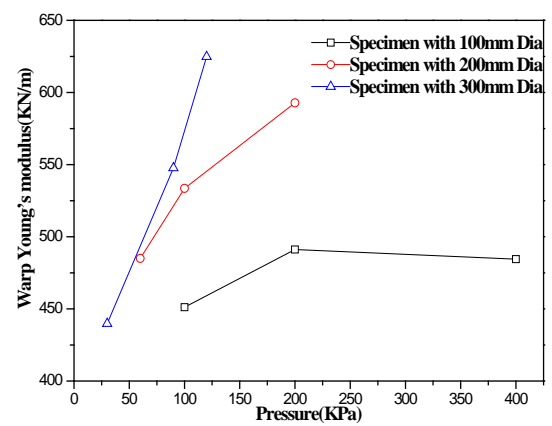

Fig. 4 Fabric modulus

Fig. 5 shows the interaction of fabric yarn contact at large pressure but not high stress level, however, material constant remains relatively stable state. Moreover, the elastic modulus was fitted to be $489.34 \mathrm{KN} / \mathrm{m}, 541.80 \mathrm{KN} / \mathrm{m}$ and $488.98 \mathrm{KN} / \mathrm{m}$ at $100 \mathrm{KPa}, 200 \mathrm{KPa}$ and $400 \mathrm{KPa}$ respectively according to the stress-strain curve of Fig. 4 for specimens, dia.100 mm, dia. $200 \mathrm{~mm}$, dia.300 mm.

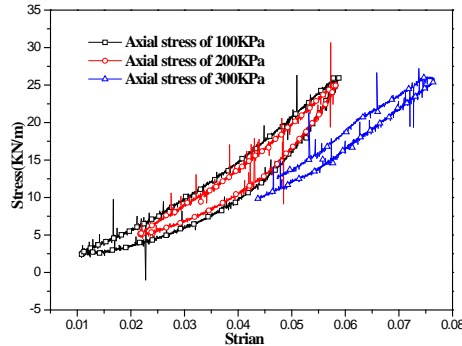

(a) $100 \mathrm{~mm}$

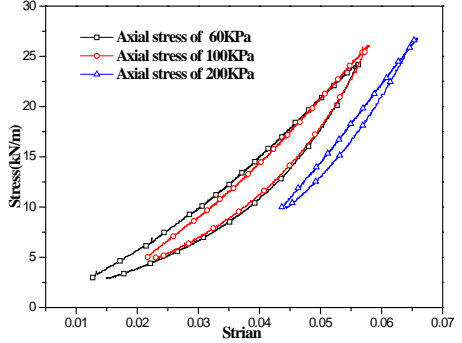

(b) $200 \mathrm{~mm}$

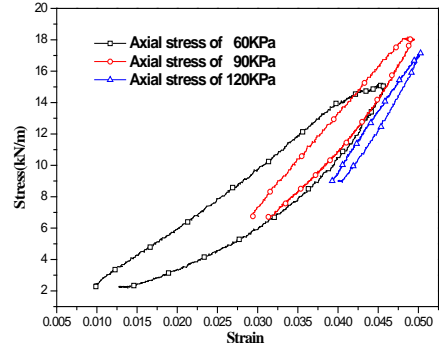

(c) $300 \mathrm{~mm}$

Fig. 5 Axial stress-strain curves of specimens with different diameters

\subsection{Tension-torsion test results analysis}

Fig. 6 suggests the relationship between applied torque and torsion angle degree, shear stress and strain of dia. $100 \mathrm{~mm}$ specimen at $100 \mathrm{KPa}$ and $200 \mathrm{KPa}$ under different axial tension. Shear modulus corresponding to $0 \mathrm{kN}, 2.5 \mathrm{kN}$ and $5 \mathrm{kN}$ is calculated to $22.02 \mathrm{kN} / \mathrm{m}, 32.68 \mathrm{kN} / \mathrm{m}$ and $41.32 \mathrm{kN} / \mathrm{m}$ severally at $100 \mathrm{kPa}$, and an increase axial force of $2.5 \mathrm{kN}$, an increase in shear modulus is $10 \mathrm{kN} / \mathrm{m}$. Corresponding shear modulus of $200 \mathrm{kPa}$ are $32.89 \mathrm{~N} / \mathrm{m}, 44.96 \mathrm{~N} / \mathrm{m}$ and $55.16 \mathrm{~N} / \mathrm{m}$, thus there is an increase of $10 \mathrm{kN} / \mathrm{m}$ of the shear modulus per $100 \mathrm{kPa}$ approximately. The above results indicate that shear stiffness of the fabric couples with the stress level of the membrane. In addition, the shear modules is estimated to $25.8 \mathrm{kN} / \mathrm{m}$ from the $1 / 20$ longitudinal modulus, less than the actual one.
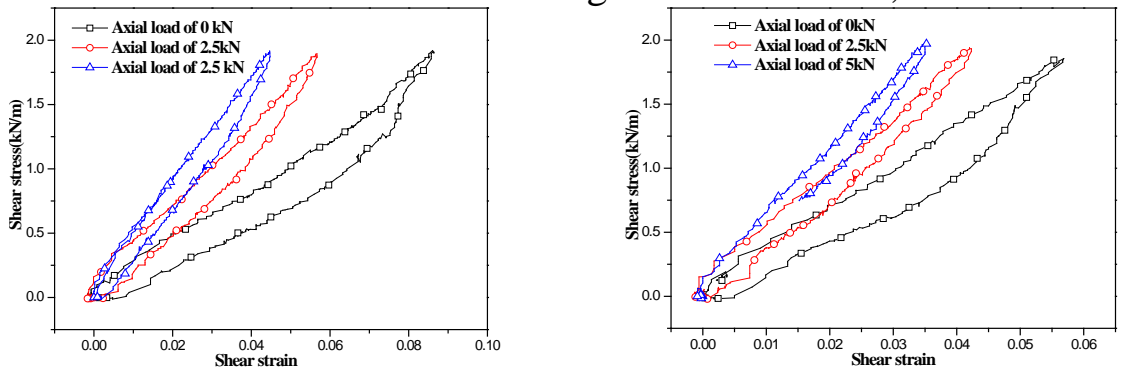

Fig. 6 Shear response of 100mm dia. specimen at 100KPa and 200KPa

\section{Derivation of scaling law of the inflatable fabric membrane}

The similarity relation of fabric membrane was deduced by dimensional analysis. The dimensionless formula of the capsule dynamics of the airbeam with the size, $l$, the density of the material, $\rho$, the elastic modulus, $E$, the pressure, $P$, Poisson's ratio, $v$, static force load, $F$, the displacement, $u$, the frequency, $\omega$, the physical similarity coefficient for the ratio of prototype and model, $\lambda$, was computed as follows:

$$
f\left(1,1,1, \frac{P}{E}, v, \frac{F}{E l^{2}}, \frac{u}{l}, \omega l \sqrt{\frac{\rho}{E}}\right)=0
$$




$$
\lambda_{P}=\lambda_{E}, \lambda_{F}=\lambda_{E} \lambda_{l}^{2}, \lambda_{u}=\lambda_{l}, \lambda_{\omega}=\frac{\lambda_{E}^{1 / 2}}{\lambda_{l} \lambda_{\rho}^{1 / 2}}
$$

The fabric film thickness reduced-scale and similar material restraints are modified using the identical material and thickness as the prototype. The similarities of the cysts are as follows:

$$
\begin{gathered}
\lambda_{t}=1, \lambda_{E}=1, \lambda_{\rho}=1 \\
\lambda_{P} \lambda_{l}=1, \lambda_{F}=\lambda_{l} \\
\lambda_{u}=\lambda_{l}, \lambda_{\omega} \lambda_{l}=1
\end{gathered}
$$

Eqs. (8)-(10) are used to design the structural parameters of the scaled model(experimental), load conditions and to predict prototype structure response or performance.

The four-point bending test was carried out to validate the theoretical deduction. The inflatable beam prototype made of QT-90S9198 polyester fiber is $5 \mathrm{~m}$ long and $0.6 \mathrm{~m}$ in diameter. The similarity coefficients of the relevant parameters are shown in Table 2:

Table 2 Inflatable beam test specimen shrinkage model parameters similarity coefficient

\begin{tabular}{|c|c|c|c|c|c|c|}
\hline Parameter & Dimension & Quality & Static load & Pressure & $\begin{array}{c}\text { Static response } \\
\text { displacement }\end{array}$ & $\begin{array}{c}\text { Natural } \\
\text { frequency }\end{array}$ \\
\hline \multirow{2}{*}{ Similarity coefficient } & $1 / 2$ & $1 / 4$ & $1 / 2$ & 2 & $1 / 2$ & 2 \\
\cline { 2 - 7 } & $1 / 4$ & $1 / 16$ & $1 / 4$ & 4 & $1 / 4$ & 4 \\
\hline
\end{tabular}

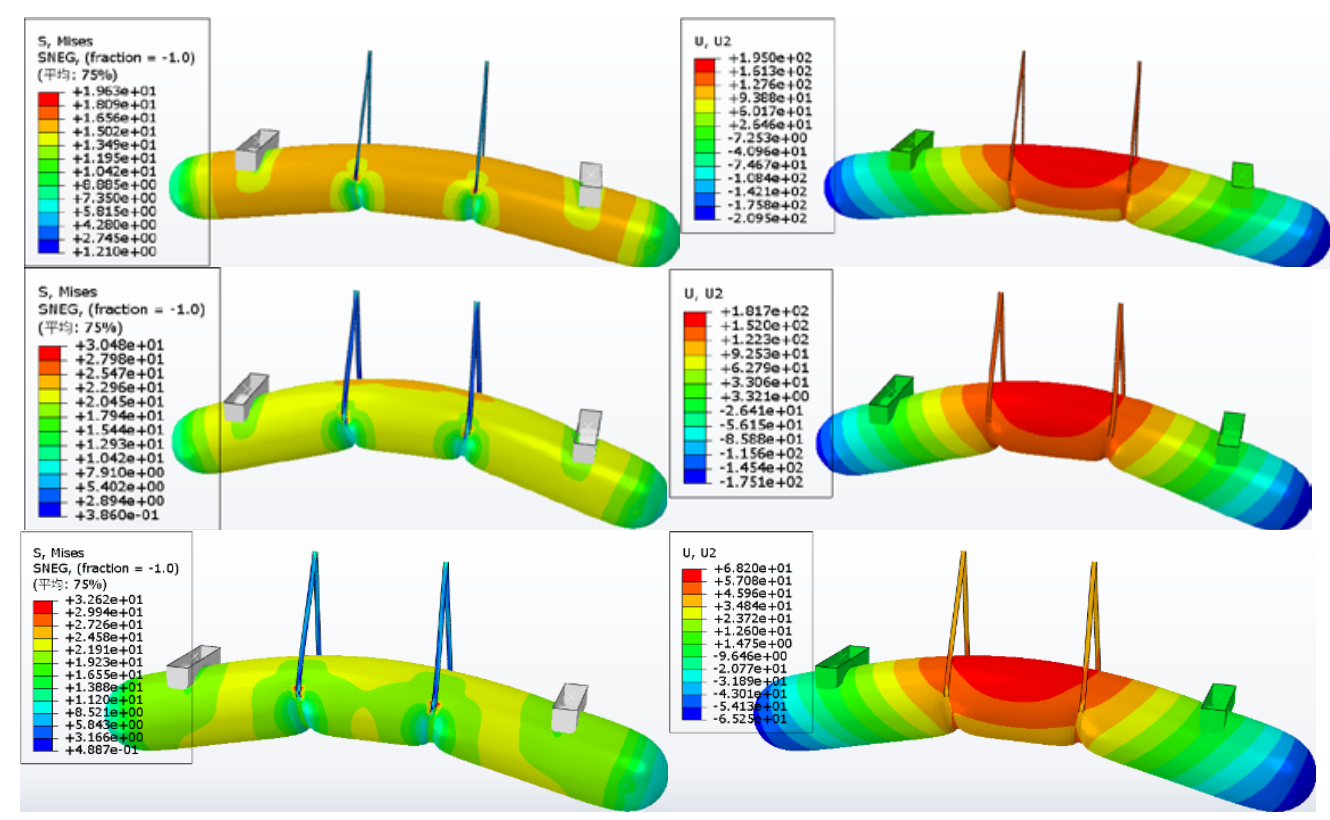

(a) Stress cloud

(b) Displacement cloud

Fig. 7 Full-size model, 1/2 scaled-model and 1/4 scaled-model

The simulation analysis of four-point bending test of the inflatable beam specimen(prototype), $1 / 2$ scaled model and $1 / 4$ scaled model were carried out, their structural displacement and stress clouds under loads of $1000 \mathrm{~N}$ and pressure of $50 \mathrm{kPa}$ were shown in Fig. 7.

The static numerical results of the $1 / 2$ scaled model and full-size specimen of the inflatable bladder are compared to calculate the scaling error, where

$$
\text { Reducted ratio error }=\frac{\text { Dis of } 1 / 2 \text { scaled model } \times 2-\text { Dis of full-scale model }}{\text { Dis of full-scale model }} \times 100 \%
$$

Fig. 8 shows that the similarity error range of node displacement is $0.5 \% \sim 7.5 \%(\leq 8 \%)$ at different internal and external pressure and load force for the1/2 scaled model; The similarity error range of each node displacement is from $-10 \%$ to $14 \%$ for the $1 / 4$ scaled model, larger than that for $1 / 2$ scaled model. 


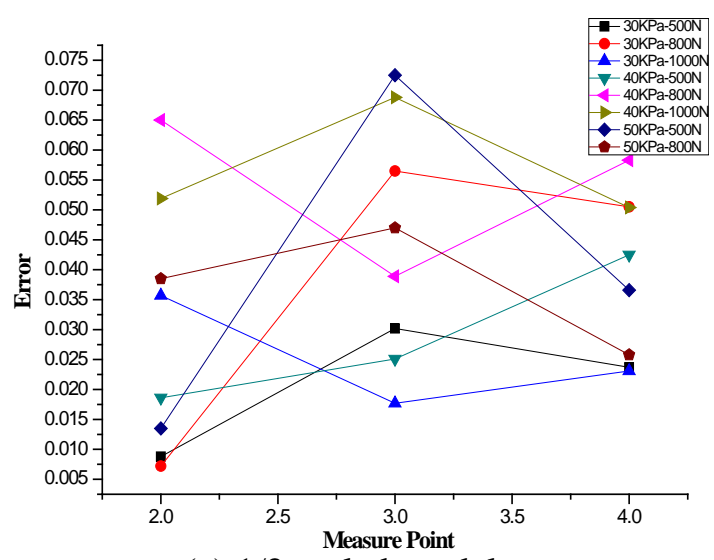

(a) $1 / 2$ scaled-model

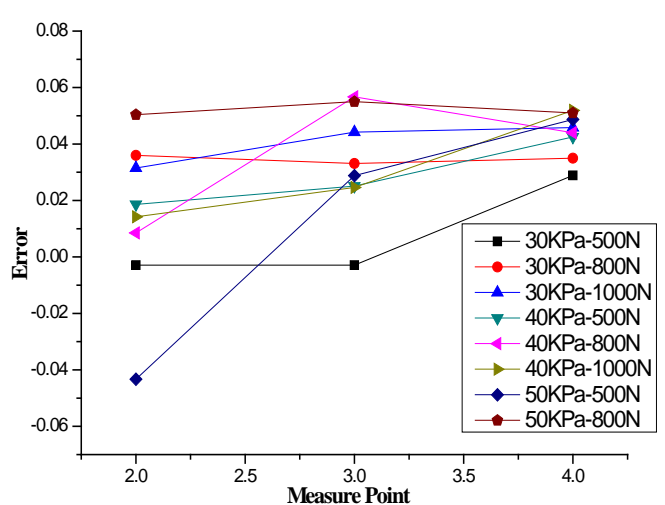

(b) $1 / 4$ scaled-model

Figure. 8 Comparison of eight operating conditions error for scaled model

\section{The scaled-model test research of inflatable fabric membrane.}

In order to validate the applicability of the scaled model design method and to evaluate the size effect on the scaled model test, a model of full-size airbeam and scaled models of 1/2 and 1/4 with length of $5000 \mathrm{~mm}, 1250 \mathrm{~mm}$ and dia. $600 \mathrm{~mm}$, dia. $150 \mathrm{~mm}$ respectively was were designed and fabricated based on simulation analysis program as shown in Figs. 9 (a)-(c).

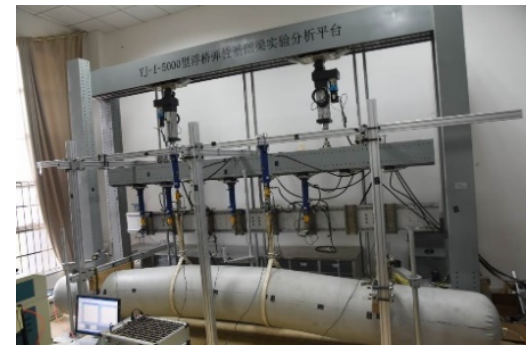

(a) Full-size model

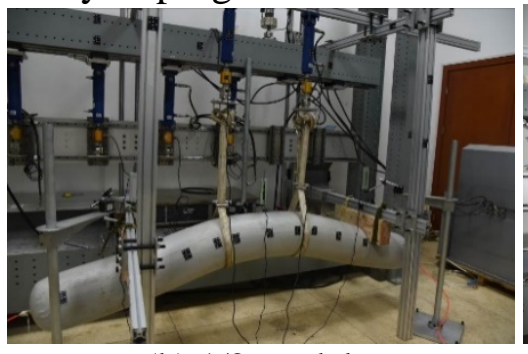

(b) $1 / 2$ model

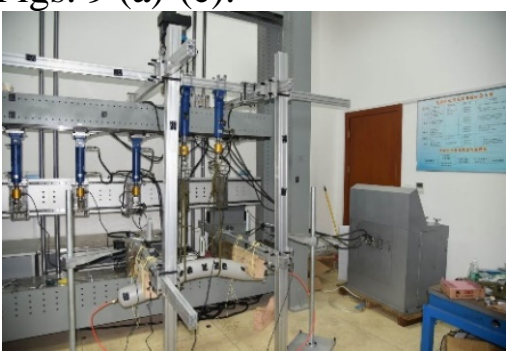

(c) $1 / 4$ model

Fig. 9 Scaled model test

Compared the numerical results of the static analysis of the $1 / 2$ scaled model of the inflatable beam specimen and the ones of the full-size specimen, and calculated scaling error, refer to Eqs.(11). The similar analysis suggests that the node displacement similar error zone is $-8 \% \sim 6 \%$ for $1 / 2$ scaled specimen at different internal and external pressure and load force, the error interval increases significantly than the ones of finite element simulation and has no obvious regularity; The similarity error range of each node displacement is $-10 \% \sim 14 \%$ for $1 / 4$ scaled model under different internal and external pressure difference and load force, the error of the middle point of the model is relatively small..

Interestingly, the comparison for $1 / 4$ and $1 / 2$ scaled model corresponding to the full-size model to predict the displacement error shows no obvious difference at $40 \mathrm{KPa}$ and the former is generally larger than the latter at 50KPa for full-scaled model. The displacement deformation error value of the full-scale airbeam specimen and the static numerical simulation of different internal and external pressure and load range is $0 \sim 10 \%$, and the calculated value is generally smaller than the experimental one.

\section{Conclusions}

Based on the testing method of elastic modulus of biaxial stretched woven fabric considering influence pressure, this paper designed an experimental tension/torsion procedure. The influence of the inflation pressure and the elastic constant of the model size material was quantified through experiments. The finite element analysis software was applied to the specimen, 1/2 scaled model and $1 / 4$ scaled model to numerically calculate response displacement of each dimension models under 
pointed load, then obtained the similarity comparison of the numerical results. The next step should consider the use of viscoelastic model for prediction; the current research only focus on the general inflatable fabric membrane materials, the study object needs to be further expanded.

\section{References}

[1] Xiao Weiwei, Chen Xujun, Fu Gongyi, Design and analysis method of outer air bag membrane tailoring for biaxial ellipsoid flexible airship Structure (2007).

[2] V.C. Paul Technology \& Mechanics Overview of Air-Inflated Fabric Structures[R]. NUWC-NPT Reprint Report 11784, December 2006.

[3] W.J. Wicker, The structural characteristics of inflatable beams. Acta Astronaut 1992;30:443-54.

[4] W.G. Davids, H. Zhang, A. Turner, M. Peterson, Beam finite-element analysis of pressurized fabric tubes. J Struct Eng, ASCE 2007;1633(7):990-8.

[5] J.P. Kabche, M.L. Peterson, W.G. Davids, Effect of inflation pressure on the constitutive response of coated woven fabrics used in airbeams[J]. Composites Part B Engineering, 2011, 42(3):526-537.

[6] G. Greschik, M.M. Mikulas, R.E. Freeland, Scale Model Testing of Nonlinear Phenomena with Emphasis on Thin Film Deployable Structures, Proceedings of the IUTAM Symposium, Solid Mechanics and its Applications, International Union of Theoretical and Applied Mechanics, Kluwer Academic, Dordrecht, The Netherlands, Vol. 80, 2000.

[7] G. Greschik, M.M. Mikulas, R.E. Freeland, Nodal Concept of Deployment and the Scale Model Testing of its Application to a Membrane Antenna, 40th AIAA/ASME/ASCE/AHS/ASC Structures, Structural Dynamics, and Materials Conference and AIAA/ASME Adaptive Structures Forum, Technical Papers, AIAA/ASME/ASCE/ AHS/ASC, Vol. 4, AIAA, pp. 2546-2554; also AIAA Paper 1999-1523, 1999.

[8] G. Greschik, Solar Sail Scalability and a “Truly Scalable” Architecture:The Space Tow. Vol. 44, No. 4, July-August 2007

[9] S. Lienard, J. Johnston, B. Ross, J. Smith, Dynamic Testing of a Subscale Sunshield for the Next Generation Space Telescope (NGST), 42nd AIAA Structures, Structural Dynamics, and Materials Conference, Seattle, WA, April 16-19, 2001, AIAA-2001-1268.

[10] S. Lienard, J. Johnston, B. Ross, J. Smith, Dynamic Testing of a One-Tenth Scale NGST Sunshield in a Vacuum Environment - Test Report, NASA Goddard Space Flight Center, February 2001.

[11] L. Xinxiang, C. Jenkins, W. Schur, Fine Scale Analysis of Wrinkled Membranes, International Journal of Computationa1l Engineering and Science, 2001.

[12] E. Jennie, Campbell*,Staged microgravity deployment of a pressurizing scale model spacecraft, 43rd AIAA/ASME/ASCE/AHS/ASC Str2uctures, Structural Dynamics, and Materials Con 22-25 April 2002, Denver, Colorado.

[13] D.M. Murphy, T. W. Murphey and P. A. Gierow, Scalable Solar- Sail Subsystem Design Concept, Journal of Spacecraft and Rockets, Vol. 40, No. 4, July-Aug. 2003, pp. 539-547.

[14] D. Lichodziejewski, B.Derbes, K. Slade, T. Mann and R. Reinert, Vacuum Deployment and Testing of a 4-Quadrant Scalable Inflatable Rigidizable Solar Sail System, 46th AIAA/ASME/ASCE/AHS/ASC Structures, Structural Dynamics, and Materials Conference and AIAA/ ASME Adaptive Structures Forum [CD-ROM] AIAA Meeting Papers on Disc, Disc 6, AIAA Paper 2005-2122, 2005.

[15] C. Galliot, R.H. Luchsinger. A simple model describing the non-linear biaxial tensile behaviour of PVC-coated polyester fabrics for use in finite element analysis[J]. Composite Structures, 2009, 90(4):438-447. 